\title{
Erythrocyte sedimentation rate and fibrinogen concentration of whole blood influences the cellular composition of platelet-rich plasma obtained from centrifugation methods
}

\author{
WENJING YIN*, ZHENGLIANG XU*, JIAGEN SHENG, XUETAO XIE and CHANGQING ZHANG \\ Department of Orthopaedic Surgery, Shanghai Jiao Tong University Affiliated Sixth People's Hospital, \\ Shanghai 200233, P.R. China
}

Received January 23, 2016; Accepted February 10, 2017

DOI: $10.3892 /$ etm.2017.4724

\begin{abstract}
Erythrocyte sedimentation rate (ESR), which reflects the sedimentation rate of platelets, leukocytes and erythrocytes in response to centrifugal force, may influence the cellular composition of platelet-rich plasma (PRP) obtained via centrifugation methods. However, no relevant studies have substantiated this. In the present study, blood was collected from 40 healthy volunteers and used to prepare PRP with two plasma-based preparation systems [YinPRP and Plasma Rich in Growth Factor (PRGF) systems] and two buffy coat-based systems (RegenPRP and WEGOPRP systems) in a single-donor model. Volumes of PRP and platelet-poor plasma (PPP) that were removed in the preparation process were recorded. Analyses of ESR, haematocrit, C-reaction protein, coagulation, serum glucose and serum lipid of the whole blood used for PRP preparation were performed to evaluate the levels of ESR and the factors known to influence it. Whole blood analysis was performed to evaluate the cellular composition of PRP. Results demonstrated that there were marked positive correlations between the ESR of the whole blood used for PRP preparation and PPP removal efficiencies, platelet concentrations, platelet capture efficiencies and platelet enrichment factors of PRP formulations obtained from plasma-based systems, and PRP yield efficiency of RegenPRP and PPP removal efficiency of WEGOPRP. Furthermore, there were marked negative correlations between ESR and concentrations and enrichment factors of platelets, leukocytes
\end{abstract}

Correspondence to: Professor Changqing Zhang or Dr Xuetao Xie, Department of Orthopaedic Surgery, Shanghai Jiao Tong University Affiliated Sixth People's Hospital, 600 Yishan Road, Shanghai 200233, P.R. China

Email: zhangcq@sjtu.edu.cn

Email: xuetaoxie@163.com

*Contributed equally

Key words: erythrocyte sedimentation rate, fibrinogen concentration, platelet-rich plasma, preparation systems, cellular composition and erythrocytes of RegenPRP. Fibrinogen concentration of the whole blood, which had a marked positive correlation with ESR, also influenced the cellular composition of PRP. These findings may increase the understanding of PRP preparation and provide substantial evidence for the individualised optimisation of PRP preparation systems used in clinical practice.

\section{Introduction}

Platelet-rich plasma (PRP) is an autologous blood product composed of concentrated platelets. $\alpha$-granules of concentrated platelets in PRP contain and release concentrated levels of platelet-derived growth factor, transforming growth factor, insulin-like growth factor and vascular endothelial growth factor, which are known to have beneficial effects on the healing process (1-3). Consequently, PRP has gained popularity in the field of tissue engineering as a growth factor delivery medium to aid in tissue healing (4-7).

Numerous PRP preparation systems are available (8-10). The common basis among these preparation systems is that leukocytes and erythrocytes will settle faster in plasma than platelets will when subjected to a centrifugal force, and therefore will become separated from the platelets (11). Hence, the sedimentation rate in response to the centrifugal force of platelets and the differences in sedimentation rates in response to the centrifugal force between platelets and erythrocytes and leukocytes are key parameters of PRP preparation systems, and may influence the cellular composition of the PRP obtained.

According to Stokes' law, the sedimentation rates of particles in a liquid environment correlate positively to the centrifugal force that the particles are exposed to (12). Thus, effort has been put into the optimization of centrifugal conditions of PRP preparation systems to maximise platelet capture efficiencies and platelet concentrations of PRP formulations obtained in an attempt to achieve the maximum therapeutic benefit $(13,14)$. However, the radius and density of particles and the density and viscosity of liquid also influence the sedimentation rates of particles, according to Stokes' law. Due to the individual differences in the radius and density of platelets, leukocytes and erythrocytes, and the density and viscosity of plasma, the optimal centrifugal condition for PRP preparation may differ individually (14). It may therefore be necessary for 
the optimisation of centrifugal conditions of PRP preparation to be individualised according to these factors. However, it is unfeasible to analyse these factors in clinical practice.

Erythrocyte sedimentation rate (ESR), a frequently used clinical laboratory test, is the sedimentation rate of erythrocytes in response to gravity $(15,16)$. Although ESR is not specific for diagnosing any disease, it is specific for the sedimentation rates of erythrocytes, leukocytes and platelets in response to the centrifugal force according to Stokes' law. Thus, ESR of the whole blood used for PRP preparation may influence the cellular composition of the PRP obtained. In this way, ESR analysis may take the place of the analyses of aforementioned factors to act as a marker of sedimentation rates of erythrocytes, leukocytes and platelets to achieve the individualised optimisation of PRP preparation. Furthermore, the levels of fibrinogen, C-reaction protein, serum glucose, serum lipid and haematocrit, which are known to influence ESR (15), may also, theoretically, influence the cellular composition of PRP; however, no relevant studies have substantiated this.

The objective of the present study was to evaluate the correlations between the cellular composition of PRP obtained from different preparation systems and the levels of ESR and factors known to influence ESR.

\section{Materials and methods}

Ethics statement. The present study was performed in accordance with the principles of the Declaration of Helsinki. The Independent Ethics Committee of Shanghai Jiao Tong University Affiliated Sixth People's Hospital (Shanghai, China) approved the protocols of the present study. Written informed consent was obtained from each volunteer.

Participant recruitment. A total of 40 healthy volunteers (31 men and 9 women) aged $41.58 \pm 9.58$ years (range, 21-59 years) were enrolled in the present study. The inclusion criterion was healthy adults who agreed to participate in the study and gave written informed consent. The exclusion criteria were a medical history of relevant diseases or consumption of any medications known to affect platelet function or concentration for 21 days before initiation of the study.

Blood collection and evaluation of whole blood characteristics. A sample of whole blood $(\sim 95 \mathrm{ml})$ was collected from each volunteer. A total of $85 \mathrm{ml}$ of whole blood was used for preparing PRP using different preparation systems in a single-donor model. The remaining blood was used as follows: Whole blood analysis was conducted using an automatic haematology analyser (XS-800i; Sysmex Corp., Kobe, Japan), ESR analysis using an automatic ESR analyser (Test 1; Alifax, Padova, Italy) and C-reaction protein analysis using an automated immunoassay system that utilised $2 \mathrm{ml}$ of blood (i-Reader; Jokoh, Shizuoka, Japan); $2.7 \mathrm{ml}$ of blood was used for coagulation analysis using an automated blood coagulation analyser (CA-7000; Sysmex Corp.); and $5 \mathrm{ml}$ of blood was used for serum glucose and lipid analyses using an automated chemistry analyser (7600-120; Hitachi, Ltd, Tokyo, Japan). All samples for clinical laboratory tests were analysed in the clinical laboratory of Shanghai Jiao Tong University Affiliated Sixth People's Hospital within 30 min of collection.
PRP preparation systems. Three commercial PRP preparation systems, approved by the Chinese Food and Drug Administration or used worldwide, and one preparation method developed in the laboratory of Shanghai Jiao Tong University Affiliated Sixth People's Hospital were used for PRP preparation. The commercial preparation systems were the Plasma Rich in Growth Factor (PRGF) system (Biotechnology Institute, Vitoria-Gasteiz, Spain), the RegenPRP system (RegenLab, Mont-sur-Lausanne, Switzerland) and the WEGOPRP system (Wego Biotechnology Co. Ltd., Weihai, China). The method developed in the laboratory of Shanghai Jiao Tong University Affiliated Sixth People's Hospital (YinPRP system) was demonstrated previously to concentrate platelets in a similar way compared with the WEGOPRP system while depleting most leukocytes and erythrocytes (data unpublished). For preparing YinPRP, $36 \mathrm{ml}$ of whole blood was anticoagulated with $4 \mathrm{ml}$ of anticoagulant citrate dextrose solution A (Shanghai Blood Centre, Shanghai, China). A total of $40 \mathrm{ml}$ of the anticoagulated whole blood was centrifuged at $160 \mathrm{xg}$ for $10 \mathrm{~min}$ at room temperature to separate platelet-containing plasma from erythrocytes and the buffy coat. Subsequently, the separated plasma was transferred to a new tube and centrifuged again at $250 \mathrm{x}$ g for $15 \mathrm{~min}$ at room temperature. After removing the supernatant of platelet-poor plasma (PPP), the precipitated platelets were resuspended in the residual plasma to obtain a total of $4 \mathrm{ml}$ of YinPRP.

According to PAW classification (17), the YinPRP and PRGF systems focus on isolating only platelets and excluding the buffy coat to remove leukocytes, and therefore are classified as plasma-based preparation systems. Contrastingly, the RegenPRP and WEGOPRP systems include the buffy coat, which contains concentrated levels of platelets and leukocytes, to capture all available platelets, and therefore are classified as buffy coat-based preparation systems. According to the centrifugation steps employed in the preparation protocols, PRGF and RegenPRP systems are classified as single-spin methods, and YinPRP and WEGOPRP systems are classified as double-spin methods.

PRP preparation. A total of $85 \mathrm{ml}$ of whole blood was divided to prepare PRP using four systems in a single-donor model to minimise the potential confounding variables $(2,8)$. The whole blood volume was divided as follows: For preparing YinPRP, $\sim 36 \mathrm{ml}$ of whole blood was used; $\sim 9 \mathrm{ml}$ for preparing PRGF; $\sim 8 \mathrm{ml}$ for preparing RegenPRP; and $\sim 32 \mathrm{ml}$ for preparing WEGOPRP. Each sample was processed simultaneously within $30 \mathrm{~min}$ of collection using the different preparation systems to prepare PRP according to the manufacturers' protocols or literature (Table I).

Evaluation of the cellular composition of PRP. The volumes of PRP and removed PPP were measured using a 1-ml micropipette (Eppendorf, Hamburg, Germany). Platelet, leukocyte and erythrocyte concentrations of PRP and whole blood were determined by whole blood analysis. PRP yield efficiency, PPP removal efficiency, platelet capture efficiency, platelet enrichment factor, leukocyte-reducing efficiency, leukocyte enrichment factor, erythrocyte-reducing efficiency and erythrocyte enrichment factor were calculated according to the formulas provided in Fig. 1. 
Table I. Protocols of the four PRP preparation systems used in the present study.

\begin{tabular}{lcccc}
\hline Protocol & YinPRP & PRGF & RegenPRP & WEGOPRP \\
\hline Net volume of WB used, ml & 36 & 9 & 8 & 32 \\
Anticoagulant (ml) & ACD-A (4.0) & ACD-A (0.9) & Sodium citrate (1.0) & Sodium citrate (3.0) \\
Number of centrifugation steps & 2 & 1 & 1 & 2 \\
Centrifugal conditions & $160 \times \mathrm{g} \times 10$ min and & $580 \times \mathrm{g} \times 8 \mathrm{~min}$ & $1,500 \times \mathrm{g} \times 9 \mathrm{~min}$ & $400 \times \mathrm{g} \times 10 \mathrm{~min}$ \\
& $250 \times \mathrm{g} \times 15 \mathrm{~min}$ & & Gel separator & and $400 \times \mathrm{g} \times 10 \mathrm{~min}$ \\
Separator system & None & None & No & No \\
Removal of buffy coat & Yes & Yes & No & Yes \\
Removal of platelet-poor plasma & Yes & Yes & &
\end{tabular}

WB, whole blood; ACD-A, anticoagulant citrate dextrose solution A; PRP, platelet-rich plasma; PRGF, plasma rich in growth factor.

$$
\begin{aligned}
& \text { PRP yield efficiency }(\%)=\frac{\text { Volume of PRP }(\mathrm{ml})}{\text { Volume of the whole blood }(\mathrm{ml})} \\
& \text { PPP removal efficiency }(\%)=\frac{\text { Volume of PPP }(\mathrm{ml})}{\text { Volume of the whole blood }(\mathrm{ml})} \\
& \text { Platelet capture efficiency }(\%)= \\
& \text { Volume of PRP }(\mathrm{ml}) \times \text { platelet concentration of PRP }\left(10^{9 / 1)}\right. \\
& \text { Volume of the whole blood }(\mathrm{ml}) \times \text { platelet concentration of the whole blood }\left(10^{9} / \mathrm{l}\right) \\
& \text { Platelet enrichment factor }(\text { fold })=\frac{\text { Platelet concentration of PRP }\left(10^{9} /\right)}{\text { Platelet concentration of the whole blood }\left(10^{9} / \mathrm{l}\right)} \\
& \text { Leukocyte-reducing efficiency }(\%)= \\
& 100 \%-\frac{\text { Volume of PRP }(\mathrm{ml}) \times \text { leukocyte concentration of PRP }\left(10^{\%} / \mathrm{l}\right)}{\text { Volume of the whole blood }(\mathrm{ml}) \times \text { leukocyte concentration of the whole blood }\left(10^{9} /\right)} \\
& \text { Leukocyte enrichment factor }(\text { fold })=\frac{\text { Leukocyte concentration of PRP }\left(10^{9} / \mathrm{l}\right)}{\text { Leukocyte concentration of the whole blood }\left(10^{9} / \mathrm{l}\right)} \\
& \text { Erythrocyte-reducing efficiency }(\%)= \\
& 100 \%-\frac{\text { Volume of PRP }(\mathrm{ml}) \times \text { erythrocyte concentration of PRP }\left(10^{12} / \mathrm{l}\right)}{\text { Volume of the whole blood }(\mathrm{ml}) \times \text { erythrocyte concentration of the whole blood }\left(10^{12} / \mathrm{l}\right)} \\
& \text { Erythrocyte enrichment factor }(\text { fold })=\frac{\text { Erythrocyte concentration of PRP }\left(10^{12} /\right)}{\text { Erythrocyte concentration of the whole blood }\left(10^{12} /\right)}
\end{aligned}
$$

Figure 1. Formulas used for the calculation of cellular characteristics of PRP. PRP, platelet-rich plasma; PPP, platelet-poor plasma.

Statistical analysis. Data were analysed using SPSS v.22.0 (IBM SPSS, Armonk, NY, USA). Kolmogorov-Smirnov tests were performed to confirm the normal distribution of continuous data. Continuous data were presented as the mean \pm standard deviation. One-way analysis of variance (ANOVA) and Bonferroni post hoc tests were performed to analyse the differences among systems. Pearson's correlation analysis was conducted to analyse linear correlations between whole blood characteristics, and between whole blood characteristics and the cellular composition of PRP. $\mathrm{P}<0.05$ was considered to indicate a statistically significant difference.

\section{Results}

Whole blood characteristics. Whole blood characteristics are demonstrated in Table II. A significantly positive correlation was observed between ESR and fibrinogen concentration $(r=0.553$; $\mathrm{P}<0.001$; Fig. 2). There was no significant correlation between ESR and the other whole blood characteristics $(\mathrm{P}>0.05)$.

Cellular composition of PRP obtained from different systems PRP yield efficiency and PPP removal efficiency. ANOVA demonstrated that there were significant differences in PRP 
Table II. Whole blood characteristics.

Characteristic

Mean \pm standard deviation

Range of normal values

Haematocrit $(\%)$

Prothrombin time (sec)

Activated partial thromboplastin time (sec)

Fibrinogen concentration (g/l)

Thrombin time (sec)

Erythrocyte sedimentation rate $(\mathrm{mm} / \mathrm{h})$

C-reactive protein $(\mathrm{mg} / \mathrm{l})$

Glucose (mmol/l)

Total cholesterol (mmol/l)

Triglyceride ( $\mathrm{mmol} / \mathrm{l})$

High-density lipoprotein cholesterol ( $\mathrm{mmol} / \mathrm{l})$

Low-density lipoprotein cholesterol ( $\mathrm{mmol} / \mathrm{l})$

Apo lipoprotein A1 (g/l)

Apo lipoprotein B (g/l)

Apo lipoprotein E (mg/dl)

Lipoprotein(a) (mg/dl)

$42.25 \pm 4.99$
$12.03 \pm 0.69$
$26.74 \pm 3.25$
$3.01 \pm 0.53$
$17.95 \pm 0.92$
$19.23 \pm 10.04$
$2.84 \pm 1.96$
$4.97 \pm 0.44$
$4.39 \pm 1.07$
$1.30 \pm 0.64$
$1.21 \pm 0.26$
$3.20 \pm 0.73$
$1.53 \pm 0.35$
$0.75 \pm 0.20$
$4.50 \pm 1.34$
$16.48 \pm 12.28$

33.50-50.80

$11.00-14.00$

20.00-40.00

2.00-4.00

13.00-21.00

$0.00-21.00$

$0.00-3.00$

$3.90-5.80$

2.80-5.90

$0.45-1.81$

$0.90-1.68$

$2.84-4.10$

$1.04-2.02$

$0.66-1.33$

$2.90-5.30$

0.00-30.00

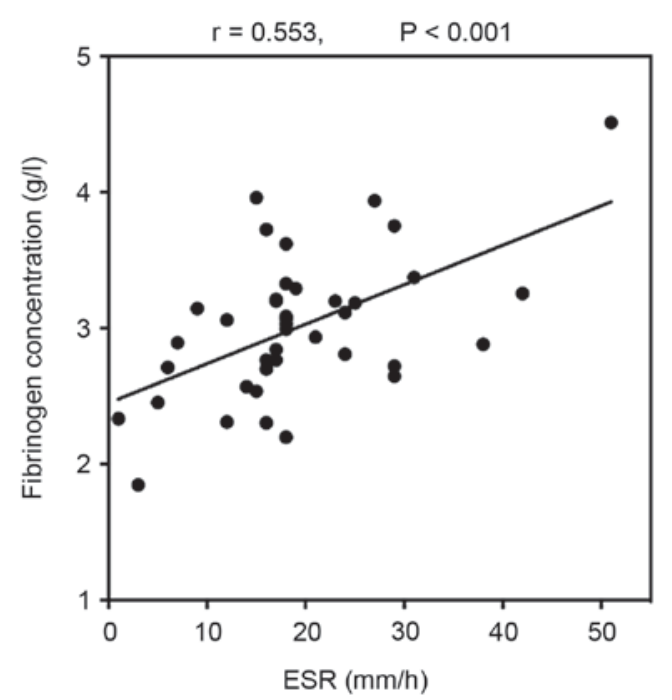

Figure 2. Significant positive correlation between ESR and fibrinogen concentration of the whole blood. ESR, erythrocyte sedimentation rate.

yield efficiency and PPP removal efficiency (both $\mathrm{P}<0.001$; Table III) among the PRP formulations obtained from the different systems. Pairwise analysis using Bonferroni post hoc testing demonstrated that PRP formulations obtained from single-spin methods (RegenPRP and PRGF) provided significantly higher PRP yield efficiencies $(\mathrm{P}<0.001)$ than PRP formulations obtained from double-spin methods (WEGOPRP and YinPRP), with RegenPRP demonstrating the highest PRP yield efficiency and YinPRP the lowest (Table III).

As the preparation protocols of the RegenPRP system did not require PPP removal (Table I), pairwise analysis demonstrated that RegenPRP had the lowest PPP removal efficiency, which was significant compared with the other systems $(\mathrm{P}<0.001$; Table III). In addition, YinPRP had a significantly higher PPP removal efficiency than PRGF ( $\mathrm{P}<0.001$; Table III).
Platelet composition of PRP. ANOVA demonstrated that there were significant differences among PRP formulations obtained from the different systems concerning platelet concentration $(\mathrm{P}<0.001)$, platelet enrichment factor $(\mathrm{P}<0.001)$ and platelet capture efficiency $(\mathrm{P}<0.001$; Table III). Pairwise analysis demonstrated that PRP formulations obtained from double-spin methods (YinPRP and WEGOPRP) had significantly higher $(\mathrm{P}<0.001$; Table III) platelet concentrations and platelet enrichment factors than PRP formulations obtained from single-spin methods (PRGF and RegenPRP). With regard to platelet capture efficiency, PRP formulations obtained from buffy coat-based systems (RegenPRP and WEGOPRP) had significantly higher platelet capture efficiencies compared with YinPRP (both $\mathrm{P}<0.001$ ), which, in turn, had a significantly higher platelet capture efficiency than PRGF $(\mathrm{P}<0.001$; Table III).

Leukocyte composition of PRP. ANOVA demonstrated that PRP formulations obtained from the different systems had significantly different leukocyte concentrations $(\mathrm{P}<0.001)$, leukocyte enrichment factors $(\mathrm{P}<0.001)$ and leukocyte-reducing efficiencies $(\mathrm{P}<0.001$; Table III). Pairwise analysis demonstrated that PRP formulations obtained from buffy coat-based systems (WEGOPRP and RegenPRP) had significantly higher leukocyte concentrations $(\mathrm{P}<0.001)$ and leukocyte enrichment factors $(\mathrm{P}<0.001)$, and significantly lower leukocyte-reducing efficiencies $(\mathrm{P}<0.001)$ than PRP formulations obtained from plasma-based systems (YinPRP and PRGF), with WEGOPRP providing the highest leukocyte concentration and leukocyte enrichment factor.

Erythrocyte composition of PRP. ANOVA demonstrated that PRP formulations obtained from the different systems differed in erythrocyte concentration $(\mathrm{P}<0.001)$, erythrocyte enrichment factors $(\mathrm{P}<0.001)$ and erythrocyte-reducing efficiencies $(\mathrm{P}<0.001$; Table III). Similar to the results of the leukocyte 


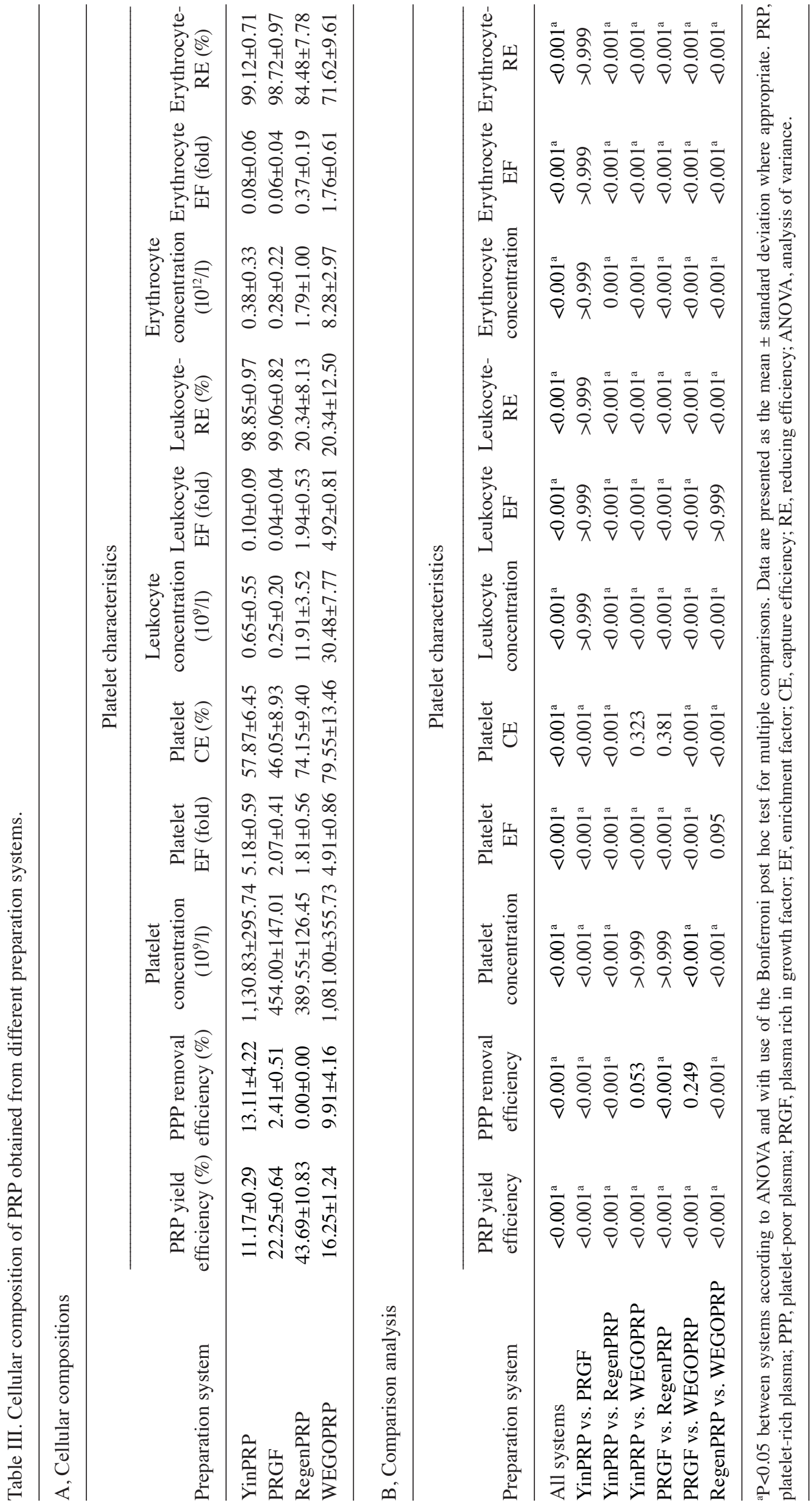




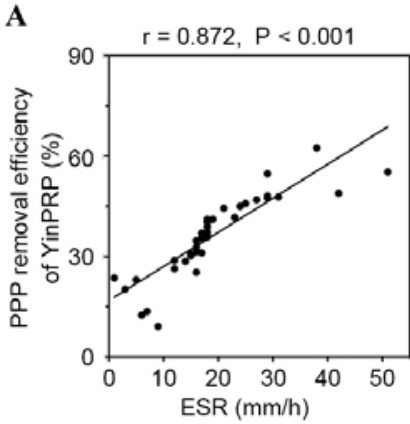

E

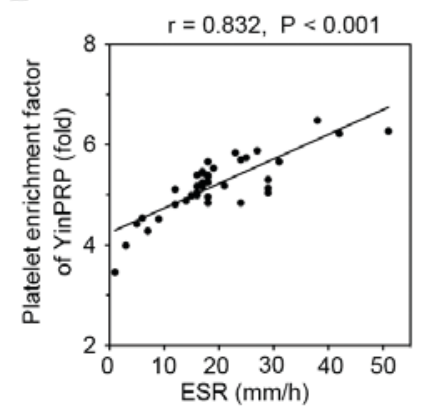

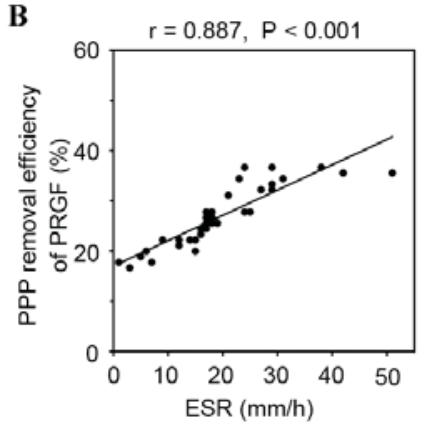

F

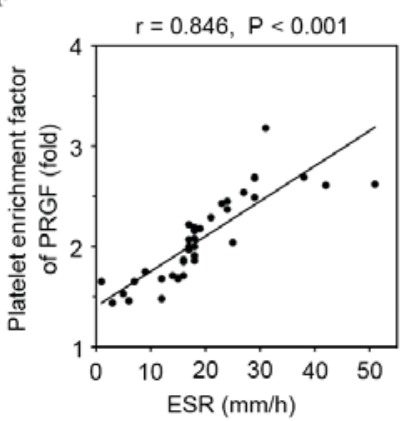

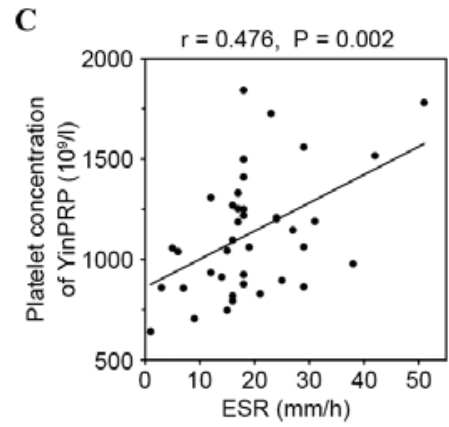

G

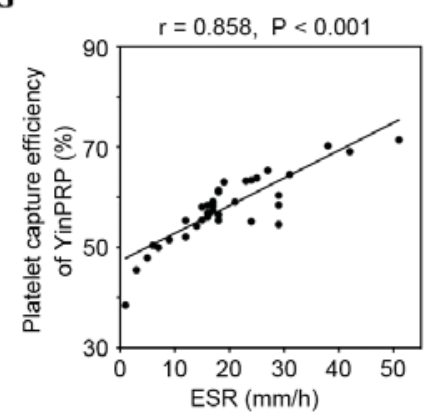

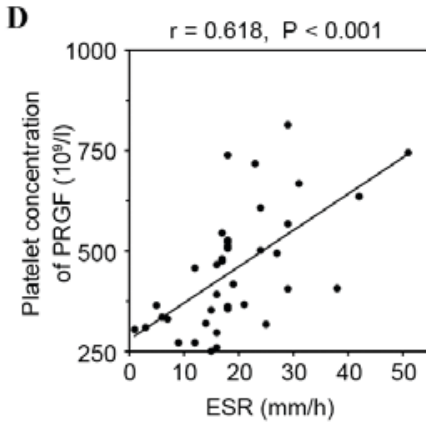

$\mathbf{H}$

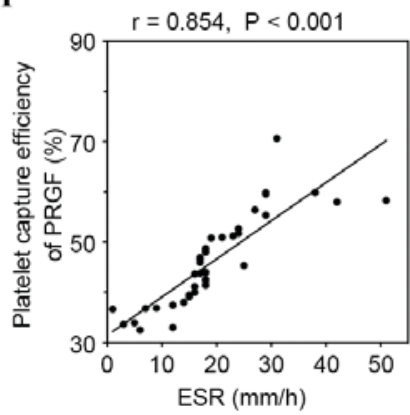

Figure 3. Significant positive correlations between ESR of the whole blood and PPP removal efficiencies of (A) YinPRP and (B) PRGF, platelet concentrations of (C) YinPRP and (D) PRGF, platelet enrichment factors of (E) YinPRP and (F) PRGF and platelet capture efficiencies of (G) YinPRP and (H) PRGF. ESR, erythrocyte sedimentation rate; PPP, platelet-poor plasma; PRP, platelet-rich plasma; PRGF, plasma rich in growth factor.

composition of PRP, pairwise analysis demonstrated that buffy coat-based PRP formulations (WEGOPRP and RegenPRP) had significantly higher erythrocyte concentrations $(\mathrm{P}<0.001)$ and erythrocyte enrichment factors $(\mathrm{P}<0.001)$, and significantly lower erythrocyte-reducing efficiencies $(\mathrm{P}<0.001)$ than plasma-based PRP formulations (YinPRP and PRGF), with WEGOPRP demonstrating the highest erythrocyte concentration and erythrocyte enrichment factor, and the lowest erythrocyte-reducing efficiency.

Correlations between whole blood characteristics and the cellular composition of PRP formulations obtained from plasma-based systems. There were significant positive correlations between ESR and PPP removal efficiencies of YinPRP $(\mathrm{r}=0.872 ; \mathrm{P}<0.001 ;$ Fig. 3A) and PRGF $(\mathrm{r}=0.887 ; \mathrm{P}<0.001$; Fig. 3B), platelet concentrations of YinPRP ( $r=0.476 ; \mathrm{P}=0.002$; Fig. 3C) and PRGF (r=0.618; $\mathrm{P}<0.001$; Fig. 3D), platelet enrichment factors of YinPRP ( $r=0.832 ; \mathrm{P}<0.001$; Fig. $3 \mathrm{E})$ and $\mathrm{PRGF}$ $(\mathrm{r}=0.846 ; \mathrm{P}<0.001$; Fig. 3F), and platelet capture efficiencies of YinPRP ( $\mathrm{r}=0.858$; $\mathrm{P}<0.001$; Fig. $3 \mathrm{G})$ and PRGF ( $\mathrm{r}=0.854$; $\mathrm{P}<0.001$; Fig. $3 \mathrm{H}$ ).

Significant positive correlations were also observed between fibrinogen concentration and PPP removal efficiencies of YinPRP ( $\mathrm{r}=0.446 ; \mathrm{P}=0.004$; Fig. 4A) and PRGF ( $r=0.419$; $\mathrm{P}=0.007$; Fig. 4B), platelet enrichment factors of YinPRP ( $\mathrm{r}=0.506 ; \mathrm{P}=0.001$; Fig. 4C) and PRGF ( $\mathrm{r}=0.420$; $\mathrm{P}=0.007$; Fig. 4D), and platelet capture efficiencies of YinPRP $(\mathrm{r}=0.560 ; \mathrm{P}<0.001$; Fig. 4E) and $\mathrm{PRGF}(\mathrm{r}=0.428, \mathrm{P}=0.006$; Fig. 4F). However, the correlations between fibrinogen concentration and platelet concentrations of YinPRP and PRGF were not significant $(\mathrm{P}=0.130$ and $\mathrm{P}=0.056$, respectively). No significant correlations between the other characteristics and the cellular composition of PRP formulations obtained from plasma-based preparation systems were demonstrated $(\mathrm{P}>0.05)$.

Correlations between whole blood characteristics and the cellular composition of PRP formulations obtained from buffy coat-based systems. Significant positive correlations were observed between PRP yield efficiency of RegenPRP and ESR ( $r=0.896 ; \mathrm{P}<0.001 ;$ Fig. 5) and fibrinogen concentration $(r=0.443 ; \mathrm{P}=0.004$; Fig. 5). However, neither ESR nor fibrinogen concentration of the whole blood influenced platelet capture efficiency $(\mathrm{P}=0.167$ and $\mathrm{P}=0.372$, respectively), leukocyte-reducing efficiency $(\mathrm{P}=0.651$ and $\mathrm{P}=0.403$, respectively) or erythrocyte-reducing efficiency of RegenPRP $(\mathrm{P}=0.363$ and $\mathrm{P}=0.799$, respectively). As a result, RegenPRP prepared using whole blood with higher levels of ESR and fibrinogen concentration was diluted by the increased PRP yield efficiency and, therefore, there were significant negative correlations between ESR and platelet concentration $(\mathrm{r}=-0.482 ; \mathrm{P}=0.002$; Fig. 6A) and enrichment factor $(\mathrm{r}=-0.633 ; \mathrm{P}<0.001$; Fig. 6B), leukocyte concentration $(\mathrm{r}=-0.642 ; \mathrm{P}<0.001$; Fig. 6C) and enrichment factor $(\mathrm{r}=-0.807$; $\mathrm{P}<0.001$; Fig. 6D), and erythrocyte concentration $(\mathrm{r}=-0.332$; $\mathrm{P}=0.037$; Fig. 6E) and enrichment factor $(\mathrm{r}=-0.323 ; \mathrm{P}=0.042$; Fig. 6F) of RegenPRP. Furthermore, there were significant negative correlations between fibrinogen and platelet concentration ( $r=-0.316$; $\mathrm{P}=0.047$; Fig. 6G), and leukocyte enrichment factor of RegenPRP ( $\mathrm{r}=-0.431 ; \mathrm{P}=0.006$; Fig. $6 \mathrm{H}$ ). However, the negative correlations between fibrinogen concentration and platelet enrichment factor $(\mathrm{r}=-0.286 ; \mathrm{P}=0.073)$, leukocyte concentration $(\mathrm{r}=-0.148 ; \mathrm{P}=0.362)$, erythrocyte concentration $(\mathrm{r}=-0.196$; $\mathrm{P}=0.226)$ and erythrocyte enrichment factor of RegenPRP $(\mathrm{r}=-0.916 ; \mathrm{P}=0.225)$ were not significant.

Similarly, the increased ESR and fibrinogen concentration of the whole blood also resulted in a significant increase in PPP 

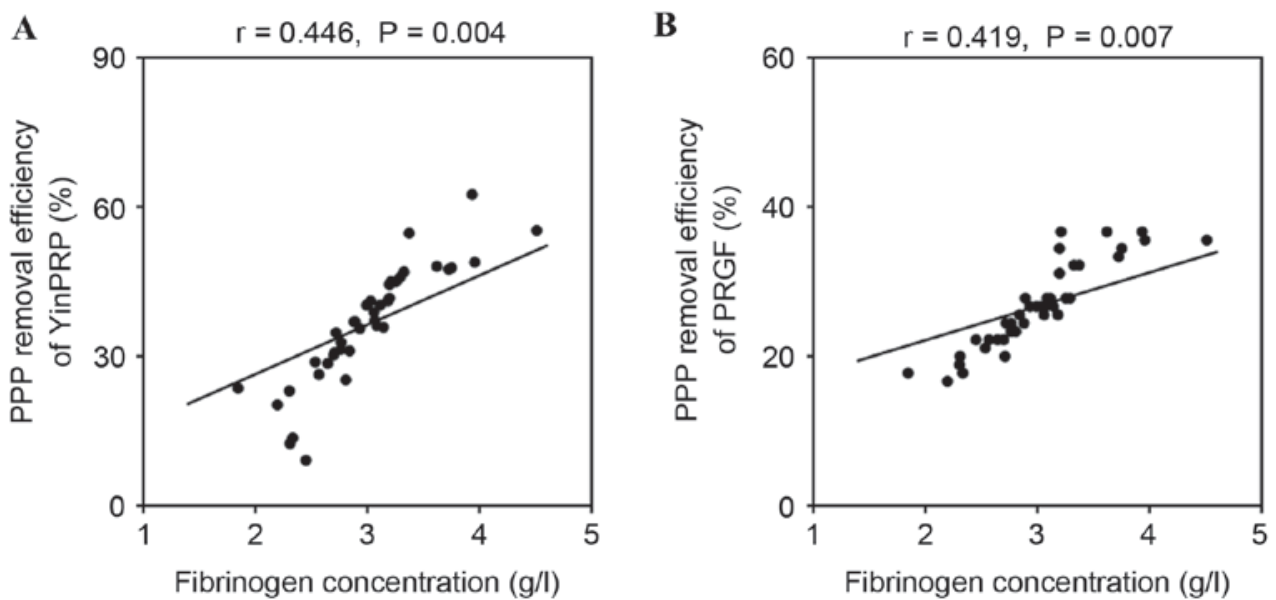

C

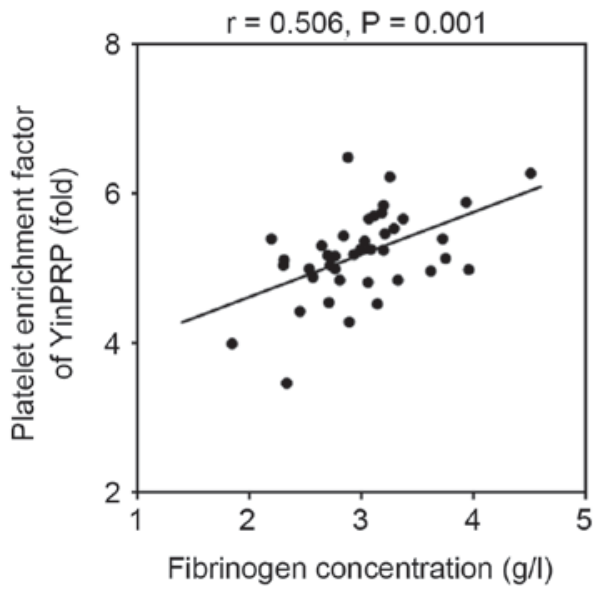

D

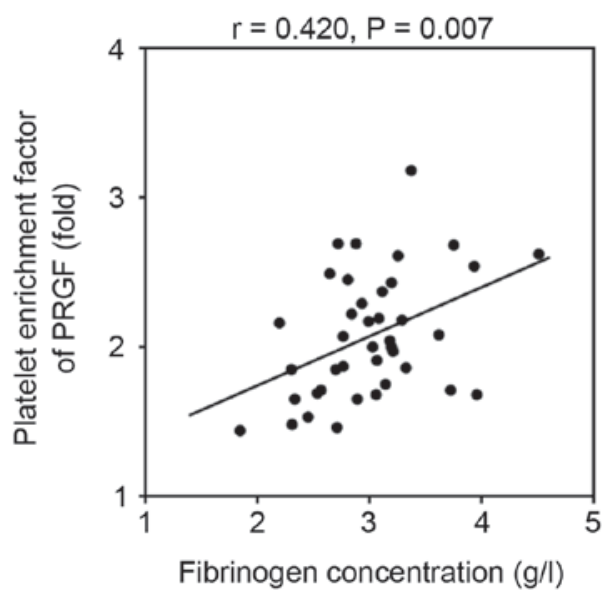

E

$\mathbf{F}$
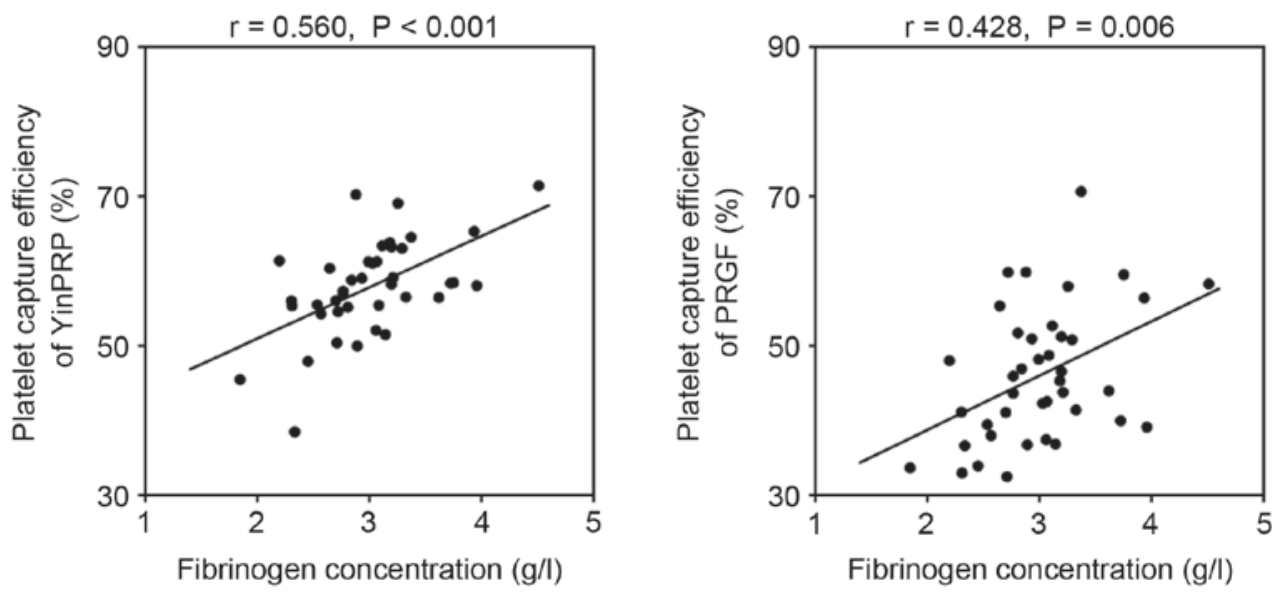

Figure 4. Significant positive correlations between fibrinogen concentration of the whole blood and PPP removal efficiencies of (A) YinPRP and (B) PRGF, platelet enrichment factors of (C) YinPRP and (D) PRGF and platelet capture efficiencies of (E) YinPRP and (F) PRGF. PPP, platelet-poor plasma; PRP, platelet-rich plasma; PRGF, plasma rich in growth factor.

removal efficiency of WEGOPRP ( $\mathrm{r}=0.900, \mathrm{P}<0.001$, Fig. 5C; and $\mathrm{r}=0.514, \mathrm{P}=0.001$, Fig. $5 \mathrm{D}$, respectively), and did not influence the platelet capture efficiency, leukocyte-reducing efficiency or erythrocyte-reducing efficiency of WEGOPRP. However, the redundant PPP was removed in the preparation process to obtain a given volume of WEGOPRP. Hence, the increased ESR and fibrinogen concentration did not result in the dilution of WEGOPRP. No significant correlations between the other whole blood characteristics and the cellular composition of PRP obtained from buffy coat-based preparation systems were demonstrated.

\section{Discussion}

The most significant finding in the present study was that ESR and fibrinogen concentration of the whole blood used for 
A

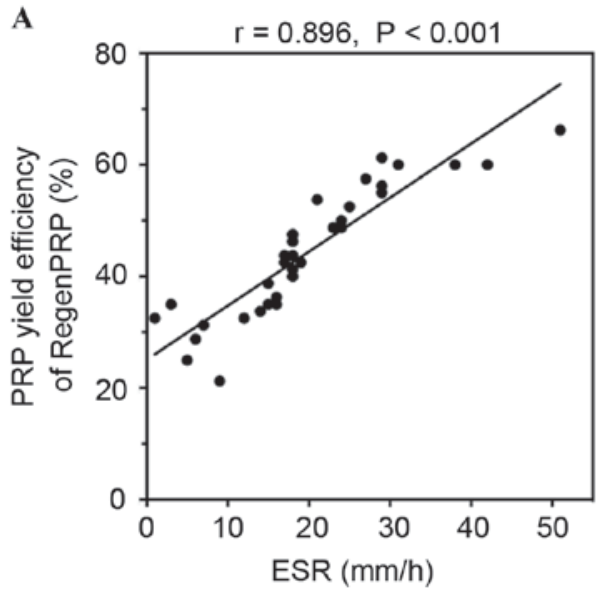

C

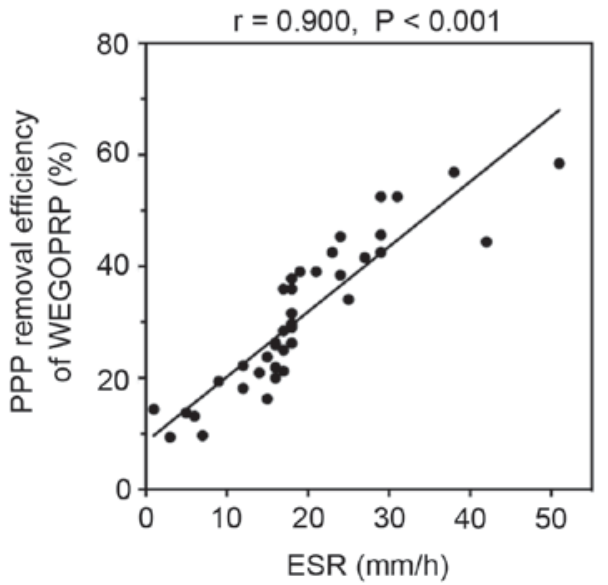

B

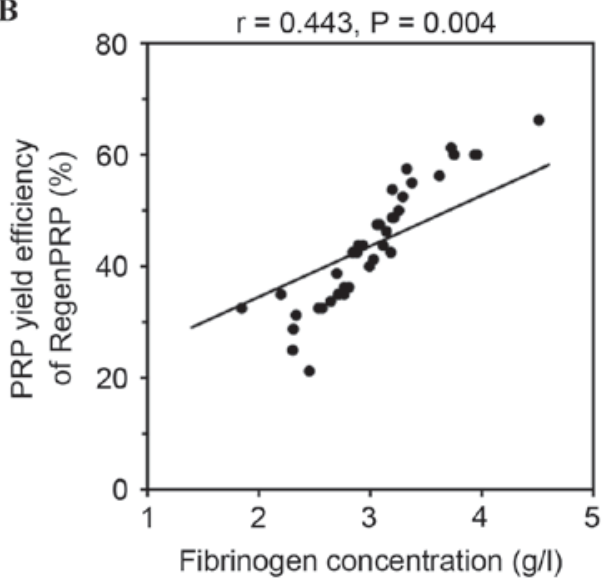

D

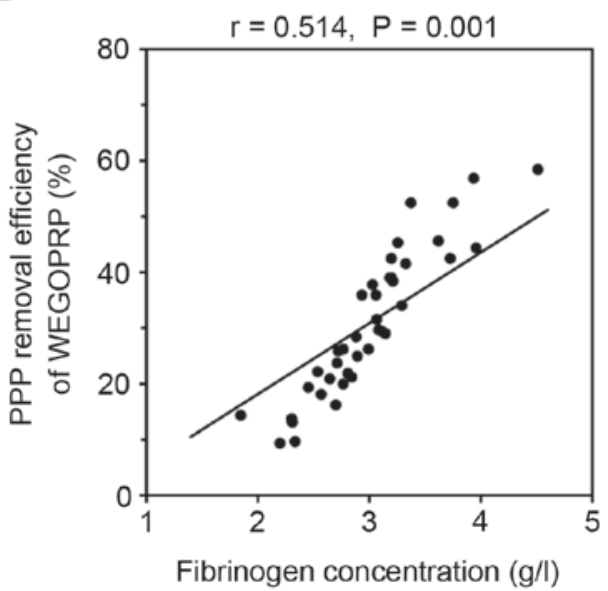

Figure 5. Significant positive correlations between the PRP yield efficiency of RegenPRP and (A) ESR and (B) fibrinogen concentration of the whole blood. Significantly positive correlations between the PPP removal efficiency of WEGOPRP and (C) ESR and (D) fibrinogen concentration of the whole blood. ESR, erythrocyte sedimentation rate; PPP, platelet-poor plasma; PRP, platelet-rich plasma.

PRP preparation influenced the cellular composition of PRP formulations obtained from plasma-based systems. To the best of our knowledge, this phenomenon has not previously been reported in literature. The possible rationale for this mechanism is that a higher fibrinogen concentration results in a higher ESR level, and a higher ESR level represents greater sedimentation rates of particles in plasma in response to the centrifugal force and greater differences in sedimentation rates between platelets and erythrocytes and leukocytes according to Stokes' law. While greater sedimentation rates of particles may result in more PPP being separated from the plasma that cells are suspended in, a greater platelet sedimentation rate may result in more platelets being separated from PPP. The greater differences in sedimentation rates between platelets and erythrocytes and leukocytes may result in more leukocytes and erythrocytes being separated from the plasma that platelets are suspended in. Hence, fewer platelets will be removed with erythrocytes, the buffy coat and PPP, and therefore more platelets will be captured and concentrated in the PRP obtained.

Nevertheless, it is not appropriate to advocate that higher ESR and fibrinogen concentration always result in higher concentrations, capture efficiencies and enrichment factors of platelets of plasma-based PRP formulations despite the centrifugal conditions. It has been demonstrated that weak centrifugal force and short centrifugal duration, which are also known as soft conditions, are not able to provide fast enough sedimentation rates of particles and great enough differences in sedimentation rates between particles for the separation of leukocytes and erythrocytes from the plasma that platelets remain suspended in $(13,14)$. However, great centrifugal force and long centrifugal duration, which are also known as hard centrifugal conditions, may result in a platelet sedimentation rate that is fast enough to separate platelets into the buffy coat at the end of the centrifugation, and therefore the optimal centrifugal conditions are required to prepare PRP $(13,14)$. High ESR and fibrinogen concentration may also result in a platelet sedimentation rate that is fast enough to separate platelets into the buffy coat, and therefore decrease the capture efficiencies, concentrations and enrichment factors of platelets of PRP formulations obtained from plasma-based methods, despite the fact that the centrifugation conditions were optimal, or even softer. Therefore, the optimal centrifugal conditions of plasma-based systems may differ according to ESR and fibrinogen concentration of the whole blood used. This indicates that the centrifugal conditions of plasma-based systems may need to be individualised according to the ESR and fibrinogen concentration of the 

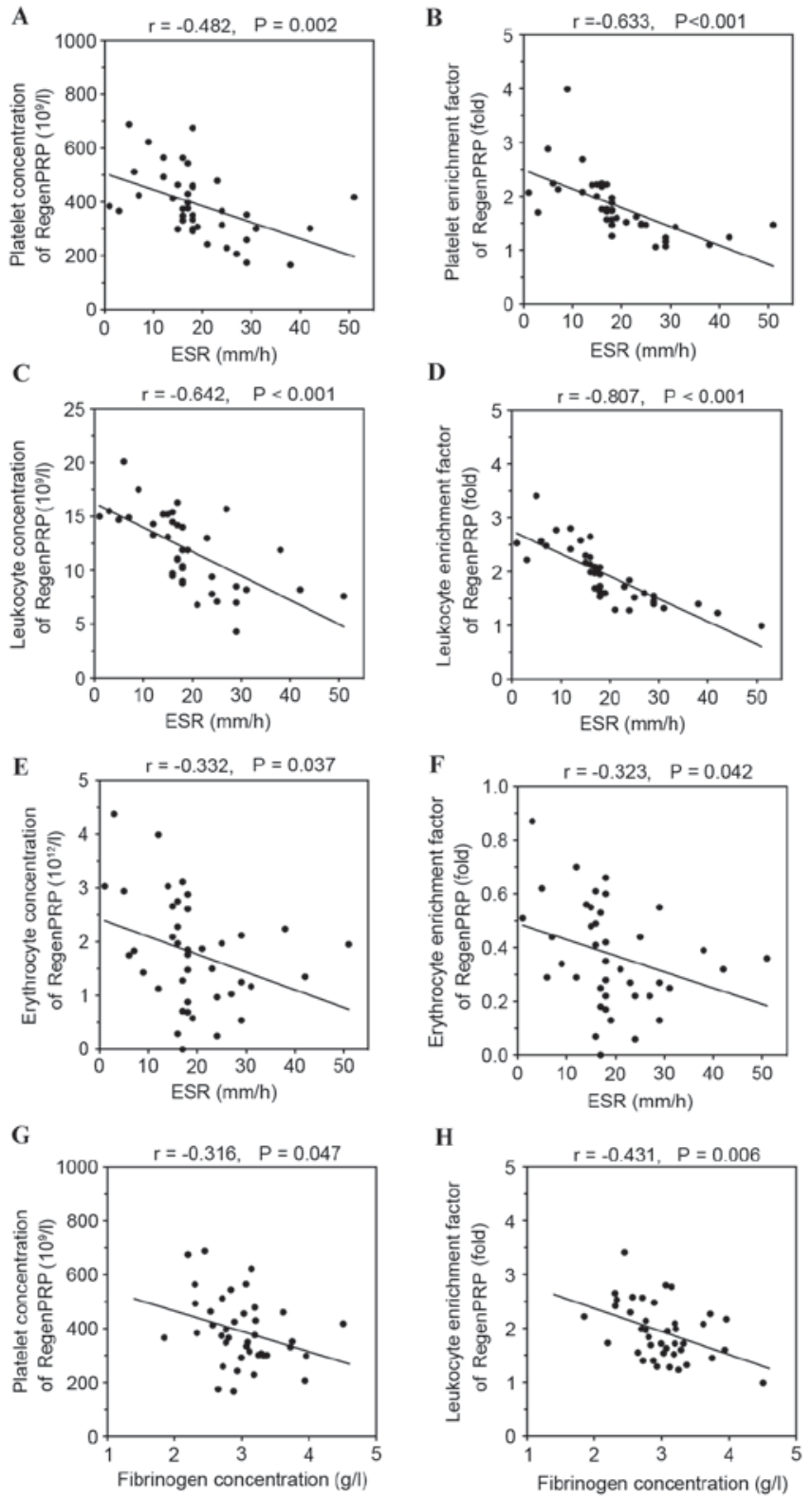

Figure 6. Significant negative correlations between ESR of the whole blood and platelet (A) concentration and (B) enrichment factor, leukocyte (C) concentration and (D) enrichment factor and erythrocyte (E) concentration and (F) enrichment factor of RegenPRP. Negative correlations between fibrinogen concentration of the whole blood and the $(G)$ platelet concentration and $(\mathrm{H})$ leukocyte enrichment factor of RegenPRP. ESR, erythrocyte sedimentation rate; PRP, platelet-rich plasma.

whole blood used to optimise the cellular composition of PRP formulations obtained.

Another notable finding of the present study was that ESR and fibrinogen concentration of the whole blood influenced the cellular composition of RegenPRP; however, they did not influence the efficiency of platelet capture, or the leukocyte- and erythrocyte-reducing efficiencies of buffy coat-based PRP formulations (RegenPRP and WEGOPRP). Due to the fact that soft centrifugal conditions may not be able to provide a fast enough platelet sedimentation rate for concentrating platelets into the buffy coat, buffy coat-based systems usually use hard centrifugal conditions in an effort to guarantee the total separation of platelets into the buffy coat at the end of centrifugation. This ensures we obtain the highest possible levels of concentrations, capture efficiencies and enrichment factors of platelets $(11,17)$. Hence, the platelet sedimentation rate in response to hard centrifugal forces used by buffy coat-based systems will remain fast enough for the total separation of platelets into the buffy coat to capture all available platelets, despite the fact that the levels of ESR and the fibrinogen concentration of the whole blood were low. However, the increased sedimentation rates of particles, represented by the increased ESR and fibrinogen concentration, did result in the higher PPP removal efficiencies. Although the redundant PPP was removed in the preparation of WEGOPRP, as well as with YinPRP and PRGF, it was not removed in the preparation of RegenPRP, and therefore, higher ESR and fibrinogen concentration resulted in the higher PRP yield efficiency of RegenPRP, and finally, resulted in the dilution of RegenPRP.

The findings of the present study suggest that the total separation of platelets into the buffy coat may have been achieved in the middle rather than at the end of centrifugation. Therefore, centrifugal conditions used by the buffy coat-based systems evaluated may be much harder than the centrifugal conditions necessary for total separation. The centrifugal conditions necessary for the total separation of platelets into the buffy coat may be softer when the whole blood with higher ESR and fibrinogen concentrations was used, and harder when the whole blood with lower ESR and fibrinogen concentrations was used. Therefore, the centrifugal conditions of buffy coat-based systems may also be individualised according to the levels of ESR and fibrinogen concentration.

Numerous studies have demonstrated that hard centrifugal conditions may be harmful to platelet function $(13,18)$. Therefore, individualised optimisation of centrifugal conditions of plasma- and buffy coat-based preparation systems may not only maximise the concentrations, capture efficiencies and enrichment factors of platelets of PRP formulations obtained. They may also soften centrifugal conditions to the necessary level to decrease the harmful effects of centrifugal conditions on platelet function. Thus, the individualised optimisation of PRP preparation may be incredibly useful for PRP therapy. Further studies with a larger sample size than the present study are required to evaluate the correlations between the ESR and fibrinogen concentration of the whole blood, centrifugation conditions used in the preparation system and the cellular composition of PRP obtained to provide substantial evidence for the individualised optimisation of PRP preparation.

A major limitation of the current study was that the volunteers were all healthy individuals with a relatively low level of ESR, while the levels of ESR of patients undertaking PRP management are usually high (15). Further studies recruiting volunteers with a relatively high level of ESR are required to evaluate the correlations identified by the present study.

In conclusion, ESR and fibrinogen concentration of the whole blood used for PRP preparation, which represent the sedimentation rates of erythrocytes, leukocytes and platelets in plasma in response to centrifugal force, are able to influence the cellular composition of PRP formulations obtained via centrifugation methods. The findings of the present study increase the understanding of PRP preparation and provide 
evidence for the individualised optimisation of PRP preparation systems used in clinical practice.

\section{Acknowledgements}

The present study was supported by the National Natural Science Foundation of China (grant no. 81401799) and Shanghai Youth Start-up Grant (grant no. 14YF1412100).

\section{References}

1. Browning SR, Weiser AM, Woolf N, Golish SR, SanGiovanni TP, Scuderi GJ, Carballo C and Hanna LS: Platelet-rich plasma increases matrix metalloproteinases in cultures of human synovial fibroblasts. J Bone Joint Surg Am 94: e1721-e1727, 2012.

2. Castillo TN, Pouliot MA, Kim HJ and Dragoo JL: Comparison of growth factor and platelet concentration from commercial platelet-rich plasma separation systems. Am J Sports Med 39: 266-271, 2011.

3. Cavallo C, Filardo G, Mariani E, Kon E, Marcacci M, Pereira Ruiz MT, Facchini A and Grigolo B: Comparison of platelet-rich plasma formulations for cartilage healing: An in vitro study. J Bone Joint Surg Am 96: 423-429, 2014.

4. Hatakeyama I, Marukawa E, Takahashi Y and Omura K: Effects of platelet-poor plasma, platelet-rich plasma, and platelet-rich fibrin on healing of extraction sockets with buccal dehiscence in dogs. Tissue Eng Part A 20: 874-882, 2014.

5. Mooren RE, Hendriks EJ, van den Beucken JJ, Merkx MA, Meijer GJ, Jansen JA and Stoelinga PJ: The effect of platelet-rich plasma in vitro on primary cells: Rat osteoblast-like cells and human endothelial cells. Tissue Eng Part A 16: 3159-3172, 2010.

6. Philippart P, Meuleman N, Stamatopoulos B, Najar M, Pieters K, De Bruyn C, Bron D and Lagneaux L: In vivo production of mesenchymal stromal cells after injection of autologous platelet-rich plasma activated by recombinant human soluble tissue factor in the bone marrow of healthy volunteers. Tissue Eng Part A 20: 160-170, 2014.
7. Bielecki T and Dohan Ehrenfest DM: Platelet-rich plasma (PRP) and platelet-rich Fibrin (PRF): Surgical adjuvants, preparations for in situ regenerative medicine and tools for tissue engineering. Curr Pharm Biotechnol 13: 1121-1130, 2012.

8. Magalon J, Bausset O, Serratrice N, Giraudo L, Aboudou H, Veran J, Magalon G, Dignat-Georges F and Sabatier F: Characterization and comparison of 5 platelet-rich plasma preparations in a single-donor model. Arthroscopy 30: 629-638, 2014.

9. Sundman EA, Cole BJ and Fortier LA: Growth factor and catabolic cytokine concentrations are influenced by the cellular composition of platelet-rich plasma. Am J Sports Med 39: 2135-2140, 2011.

10. Mazzocca AD, McCarthy MB, Chowaniec DM, Cote MP, Romeo AA, Bradley JP, Arciero RA and Beitzel K: Platelet-rich plasma differs according to preparation method and human variability. J Bone Joint Surg Am 94: 308-316, 2012.

11. Arnoczky SP and Sheibani-Rad S: The basic science of platelet-rich plasma (PRP): What clinicians need to know. Sports Med Arthrosc 21: 180-185, 2013.

12. Stokes GG: On some cases of fluid motion. Trans Camb Philos Soc 8: 105-165, 1843.

13. Bausset O, Giraudo L, Veran J, Magalon J, Coudreuse JM, Magalon G, Dubois C, Serratrice N, Dignat-George F and Sabatier F: Formulation and storage of platelet-rich plasma homemade product. Biores Open Access 1: 115-123, 2012.

14. Araki J, Jona M, Eto H, Aoi N, Kato H, Suga H, Doi K, Yatomi Y and Yoshimura K: Optimized preparation method of platelet-concentrated plasma and noncoagulating platelet-derived factor concentrates: Maximization of platelet concentration and removal of fibrinogen. Tissue Eng Part C Methods 18: 176-185, 2012.

15. Schulak DJ, Rayhack JM, Lippert FG III and Convery FR: The erythrocyte sedimentation rate in orthopaedic patients. Clin Orthop Relat Res: 197-202, 1982.

16. Covey DC and Albright JA: Clinical significance of the erythrocyte sedimentation rate in orthopaedic surgery. J Bone Joint Surg Am 69: 148-151, 1987.

17. DeLong JM, Russell RP and Mazzocca AD: Platelet-rich plasma: The PAW classification system. Arthroscopy 28: 998-1009, 2012.

18. Dohan Ehrenfest DM, Rasmusson L and Albrektsson T: Classification of platelet concentrates: From pure platelet-rich plasma (P-PRP) to leucocyte- and platelet-rich fibrin (L-PRF). Trends Biotechnol 27: 158-167, 2009. 